Lexis Vol. XLI (2) 2017: 427-454

\title{
Objeto y materia de estudio de la lingüística. A propósito de la toponimia y sus métodos
}

\author{
Luis Fernando Rubio \\ Pontificia Universidad Católica del Perú
}

\begin{abstract}
RESUMEN
El tema que le interesa a este trabajo está relacionado con el aspecto teórico del quehacer lingüístico. Le importa destacar su relevancia, en especial, cuando se trata de una disciplina que trabaja sobre un objeto como la lengua, producto mismo de la reflexión teórica. Para esto, se propone un ejercicio metateórico en el que se distinguen el objeto de estudio de la materia de una subdisciplina de la lingüística como la toponimia. El objetivo es demostrar cómo, desde la supuesta homogeneidad de una misma área de interés, el objeto y la materia de estudio con que se suele trabajar es, en realidad, el resultado de una elaboración teórica y metodológica guiada por los intereses y objetivos particulares del investigador.
\end{abstract}

Palabras clave: onomástica, toponimia, hermenéutica, epistemología, objeto y materia de estudio

\section{ABSTRACT}

The following paper is interested in the theoretical aspect of linguistic work. That is why it points out its importance when dealing with disciplines such as linguistics, which work on objects that are the product of theoretical reflections. For this purpose, we try to distinguish which are the different 
objects of study used in a field of linguistics such as toponymy. The main objective is to demonstrate how the objects used for linguistic analysis are, in reality, the result of a theoretical and methodological elaboration guided by the particular interests and objectives of the researcher

Keywords: onomastics, place names, hermeneutics, epistemology, object of study

Las siguientes páginas contienen algunas reflexiones acerca del objeto material y el objeto formal de la toponimia, y tienen como principal finalidad demostrar cómo es que alrededor de esta rama de la onomástica, dedicada al estudio de los nombres que una comunidad de habla utiliza (o utilizó) para referirse a los lugares dentro del espacio geográfico donde habita, se han construido diferentes objetos de estudio de acuerdo con las perspectivas y objetivos de determinadas subdisciplinas de la lingüística. El tipo de análisis metateórico que aquí se propone no es un ejercicio que debiera resultar ajeno a la lingüística, sobre todo, al ser una disciplina que pretende estudiar un fenómeno tan amplio y todavía desconocido como el lenguaje. De hecho, puede que la variedad de ramas que han aparecido (y que aparecen) dentro de la disciplina sea un indicio suficiente para hacer válida la pregunta acerca del rumbo que la lingüística ha tomado y, claro, para preguntarse si las metodologías que se han creado hasta aquí coinciden o no con sus objetivos. De modo que el interés de este trabajo es brindar un ejemplo acerca de cómo, desde el caso particular de la toponimia, el paso de la materia al objeto se lleva a cabo gracias a un proceso de selección (de un fenómeno particular de la realidad) y construcción (de un conocimiento determinado).Para esto, debe tomarse en cuenta que un objeto de estudio es el resultado, siempre, de una construcción puntual, de una manera particular de plantearse la observación de un fenómeno específico de la realidad. Así, en primer lugar, se llevará a cabo una breve descripción del contraste entre el objeto material (materia) y objeto formal (objeto de estudio) como parte del desarrollo de la lingüística y cualquier otra disciplina científica. Esta 
distinción es fundamental para entender cuál es la realidad objetiva del lenguaje que le interesa al lingüista y qué actos epistémicos del conocimiento son los que debe tomar en cuenta para aproximársele. Ahora bien, es aquí que las ideas de Itkonen (2008) —basadas en la concepción popperiana de que existen diversos niveles ontológicos que construyen la realidad, y son los conceptos científicos los que proporcionan conocimiento sobre la misma- servirán de apoyo para determinar una relación entre la materia, el objeto, la metodología y los objetivos de cualquier actividad de investigación lingüística.

Con este propósito, se pretender analizar y explicar las finalidades, metodología y objeto de estudio con que trabajaron tres investigaciones disímiles referidas al campo de la toponimia: Columbia Plateau Indian Place Names: What can they teach us? (Eugene Hunn 1996), Morfemas arcaicos en la toponimia centroandina: los sufijos $-y,-n$ y $-r a$ (Cerrón-Palomino 2008c) y Toponimia sevillana. Ribera, Sierra y Aljarafe (Gordón 1995). Si bien en apariencia estos textos se enfocan en una misma o similar materia de estudio, en realidad, parten de distintas tradiciones e intentan cumplir con objetivos diferentes. Es en este sentido que, mediante su análisis, se espera entender el proceso, las semejanzas y diferencias con las que se construyen distintos objetos de estudio, guiados siempre por enfoques y necesidades particulares.

\section{Materia y objeto}

Toda disciplina científica $-\mathrm{y}$ sus distintas ramas- enfoca sus esfuerzos y objetivos alrededor de un objeto de interés. Este se constituye según la relación entre una realidad u objeto material (materia) y un objeto formal (objeto de estudio). La primera puede ser compartida con otras disciplinas, mientras que el segundo debería ser característico y propio de cada una. Entender la diferencia entre la materia y el objeto de estudio (determinado gracias a una construcción teórica) (López Serena 2013: 78) resulta de gran importancia al momento de plantear la metodología más adecuada 
para llevar a cabo un análisis coherente. De esta manera, cuando se habla de materia, se puede pensar en todos los hechos ocurridos dentro de una realidad concebida, teniendo en cuenta que lo entendido como real no es únicamente físico o accesible mediante la observación, sino que, también, comprende una serie de conocimientos de carácter psicológico y/o social. El objeto, por otro lado, es el producto de las perspectivas teóricas y las finalidades con que se observa esta realidad. Sus características serán, más bien, el resultado de un tipo de acceso particular a la materia de estudio, sea mediante el uso de los sentidos o la reflexión con respecto a un tipo de saber específico. En lo que refiere a las humanidades, la imposibilidad que se tiene de acceder a la materia de estudio a través de la observación exige preguntarse cómo es que se comprenden los distintos fenómenos de la realidad.

¿Cómo se concibe, entonces, de manera objetiva la materia? Como explica Henríquez Garrido (2004), durante la primera década del siglo XX, el conductismo se afianzó como la postura científica oficial ante la explicación de los fenómenos mentales. Postura que pretendía explicar la conducta humana únicamente a partir de los aspectos empíricamente observables. Sin embargo, como Itkonen señala (2008: 91), todos los intentos del materialismo fisicalista por demostrar esta reducción fracasaron, quizás debido a la dificultad que supone describir un fenómeno mental sin el apoyo de una terminología que contemple tales aspectos, como la del dualismo. ${ }^{1}$ En este sentido, en tanto que la observación solo supone una de las herramientas de aproximación a la realidad, específicamente a los

1 El dualismo, dicho de manera resumida, supuso una respuesta al materialismo fisicalista debido a que consideraba lo mental como un fenómeno no físico de carácter interno. No obstante, como explica Henríquez Garrido (2004), existen algunos elementos en su contra, "generalmente es aceptado que todo fenómeno natural está constituido sobre fundamentos físicos elementales. No obstante, el dualismo, define la mente fuera de esta representación física, lo que le sitúa en una posición muy incómoda a la hora de estudiar lo mental con los métodos científicos usuales. Otra dificultad que ofrece el dualismo es el problema de las relaciones cuerpo-mente: ¿cómo explica que una sustancia o entidad no física como la mente pueda tener efectos sobre la materia, provocar acciones, es decir, interactuar casualmente con una entidad física como el cuerpo?” (2004: 116-117). 
hechos materiales, es necesario contemplar un modelo que permita entender el modo en que se conciben y construyen realidades desde la mente, y de qué manera los conceptos científicos suponen un apoyo para indagar dentro de la misma.

En las últimas décadas, parece haberse dado una suerte de consenso entre lingüistas acerca de las características necesarias para que la disciplina sea considerada científica. Aparentemente, ese acuerdo ha tenido como base más sólida el contar con evidencia empírica capaz de sostener la objetividad de cualquier análisis o investigación. No obstante, al momento de definir qué es un dato empírico, generalmente, se ha aceptado que debe tener como principal característica la posibilidad de ser falsado a través de una observación realizada en el espacio-tiempo. Ahora bien, más allá de que algunas subdisciplinas de la lingüística basen sus resultados en datos concretos del habla que, en efecto, tienen un lugar en el tiempo y el espacio, las conclusiones y metas a las que apuntan suponen una síntesis de esos datos. En otras palabras, el objeto de conocimiento que interesa no es el dato, sino las reglas detrás de él. Dicho de otro modo, aunque para aprender una lengua (y estudiarla) se necesita de la observación, del habla, el conocimiento de la misma se construye como un hecho de conciencia. Debido a esto, aunque necesario, el dato empírico solo debe ser visto como un instrumento, lo cual hace de la observación un acto epistémico incapaz de ahondar dentro de este tipo de conocimiento, pues pertenece a otro nivel. Para acceder al saber lingüístico, resulta necesario un modelo epistemológico que contemple la existencia de diferentes realidades construidas por la mente. De manera que, en salvedad del ejercicio que en este trabajo se plantea, se ha decidido tomar en cuenta la perspectiva de Itkonen (2008), quien utiliza el modelo de niveles ontológicos planteado por Popper para el análisis conceptual de las realidades humanas. ${ }^{2}$

2 El planteamiento de diversos niveles ontológicos que permiten entender de manera más adecuada cómo se elabora el conocimiento, y cuál es la forma de la realidad natural y social, no es exclusivo de Popper. Por ejemplo, el propio Itkonen señala que "Bechtel y Abrahamsen (1992, 256-261) defienden la existencia de cuatro 
El razonamiento de Itkonen pretende explicar cómo es permisible la aproximación a los hechos del lenguaje desde actividades cognitivas distintas de la observación, específicamente la intuición y la introspección. Ambas aproximaciones contemplan diversos tipos de conocimientos mentales, tanto intersubjetivos como subjetivos, a saber, realidades diferentes de lo estrictamente material. Por este motivo, sobre la base de un modelo popperiano del aprendizaje a partir de tres mundos del conocimiento, Itkonen intenta explicar cuáles son los lugares que ocupa el lenguaje y cómo condicionan la labor del lingüista.

En este contexto, yo aceptaré la tripartición ontológica de Popper (1972), es decir, la que divide la ontología en los «mundos»: (i) de los estados y hechos físicos, (ii) de los estados y hechos psicológicos y (iii) de los conceptos y las normas sociales. Las etiquetas que sirven para designar estos mundos son «m-1», «m-2»y «m-3». Cada uno de ellos requiere su propio acto epistémico característico: $\mathrm{m}-1$ = la observación; $\mathrm{m}-2$ = la introspección; $\mathrm{m}-3$ = la intuición. De las tres, la observación es la única que está basada en el empleo de uno de los cinco sentidos (Itkonen, 1981b). (Itkonen 2008: 91)

El modelo de comprensión del conocimiento que le interesa a Itkonen pretende explicar que todo cuanto existe se determina en tres mundos. El mundo $1(\mathrm{~m}-1)$ comprende aquello que se adquiere y se controla a través de los sentidos. El mundo $2(\mathrm{~m}-2)$ abarca todo los fenómenos propios de la mente, concebidos a partir de criterios subjetivos individuales que todavía no han sido evaluados socialmente. El mundo $3(\mathrm{~m}-3)$ posee la base de todo conocimiento, aquí se encuentran la cultura, el arte y la ciencia. Itkonen (2008) señala que "los tres tipos de actos son potencialmente conscientes y, en tanto que actos psicológicos subjetivos, todos emanan del m-2” (91).

niveles, que ellos denominan, de acuerdo con las disciplinas de estudio correspondientes: ciencias físicas < ciencias biológicas < ciencias del comportamiento (incluida la psicolingüística) < ciencias de la cultura (incluidas la lingüística y la lógica)" (2008: 91). Debe quedar claro, entonces, que el uso del modelo popperiano por parte de Itkonen seguramente se debe a que gran parte de la filosofía de Karl Popper se centró en la crítica de las refutaciones empíricas de las teorías científicas. 
En efecto, existe una interacción entre estos mundos, pues la observación de las realidades físicas y teóricas se lleva a cabo como un acto individual desde m-2 sobre el m-1 y m-3, ambos autónomos y únicos para todos los seres humanos. La hipótesis popperiana de un tercer mundo "invierte el objeto de conocimiento de todas aquellas ciencias que pretenden estudiar la mente [entre ellas la lingüística], partiendo de los efectos que le son propios, esto es, de los productos mentales, como las teorías, los problemas y el conocimiento objetivo en general” (Henríquez Garrido 2004: 119). En otras palabras, quiere explicar cómo es que los fenómenos de la mente pueden tener efectos sobre la materia y el modo en que se la comprende intersubjetivamente.

Dicho esto, resta explicar en líneas generales cómo se construye el objeto de estudio de la lingüística a partir del modelo de los tres mundos, por lo menos desde una perspectiva hermenéutica. Como señala Fernández Pérez (1986), la multiplicidad de facetas que ofrece el lenguaje ha permitido que un conjunto de subdisciplinas de la lingüística lo observen con intereses y finalidades distintas. Esta misma autora señala que, para Itkonen, los fenómenos lingüísticos se convierten en datos de investigación siempre que sean observados según su valor intersubjetivo, o sea, según un criterio de norma o conocimiento social que corresponda al m-3. Por tanto, se puede entender que "la investigación de estos datos está ligada a la reflexión, y de ahí la inclusión de la lingüística en el campo de la hermenéutica" (Fernández Pérez 1986: 34). Cuando una disciplina, como la que aquí se discute, no posee, única y exclusivamente, un acceso material a su objeto, puede enfocar su interés en un tipo de conocimiento que forme parte de la realidad conceptual e intersubjetiva $(\mathrm{m}-3)$ o, también, en un conocimiento individual y subjetivo $(\mathrm{m}-2)$. Ahora bien, cuando se intenta describir un tipo de conocimiento social, la recolección de datos se realiza de manera intuitiva y supone, además, un primer acercamiento a un objeto de estudio ajeno al investigador. Por el contrario, si el interés está en describir un tipo de realidad individual y psicológica, inaprensible por observación o intuición, acercarse al objeto implica ir detrás de estas 
realidades psicológicas mediante inferencias a partir de datos en un corpus cualquiera.

Cuando hay una razón para hablar del objeto de investigación como algo distinto de los datos y subyacentes a éstos, entonces el objeto de investigación siempre es hipotético o incierto. Un ejemplo de esto lo constituyen las estructuras y/o los procesos psicológicos inconscientes que se investigan (es decir, sobre los que se emiten hipótesis) en función del comportamiento lingüístico observable y/o sobre la base de la intuición consciente. En este caso, los procesos y/o estructuras constituyen el objeto de investigación, mientras que el comportamiento y las intuiciones lingüísticas constituyen los datos. (Itkonen 2008: 80)

La idea es que si un campo de estudio no tiene acceso al objeto de su interés mediante la observación o la intuición, entonces, sigue de ello que, en efecto, la naturaleza del estudio es hipotética. A la lingüística, al menos la que describe Itkonen, le interesan especialmente los procesos sistemáticos vistos como una norma, es decir, conocer cómo operan y qué son precisamente las estructuras intersubjetivas que rigen una lengua. Así, la recolección de datos es el resultado de una interacción con la materia y, a la vez, el modo de acceso al objeto de estudio. Los comportamientos lingüísticos seleccionados serán pertinentes, sin importar su tipo de materialidad -escrita o fónica-, siempre que sean representativos del objeto y permitan cumplir con los principales objetivos planteados. A fin de cuentas, las distintas disciplinas científicas (y las ramas afines a cada una de estas) se deben distinguir unas de otras, fundamentalmente, por un objeto de estudio delimitado, de modo que se debería poder encontrar tantas (disciplinas y ramas) como objetos formales existan.

\section{Análisis de los casos}

En las siguientes páginas se intentará especificar la diferencia entre el objeto de estudio y la materia de tres perspectivas de análisis distintas de la toponimia: los trabajos de Cerrón-Palomino (2008c), 
Gordón (2005) y Hunn (1996), ya mencionados algunas páginas atrás. Desde ya, es posible decir que, aunque cada uno de estos casos comparte la misma materia de estudio - o sea, la toponimia vista como el conjunto de nombres de lugar con que una comunidad se refiere al espacio geográfico que le pertenece-, se espera demostrar cómo sus distintas perspectivas teóricas influyen en la manera como se observa la materia y cómo esto contribuye, por tanto, al planteamiento de un objeto y una metodología distintas. Con este propósito, primero, se procurará distinguir entre la materia y objeto de estudio de cada uno de estos textos para, luego, describir su metodología y discutir si es la más conveniente para el objeto conceptualizado.

\subsection{Toponimia sevillana. Ribera, Sierra y Aljarafe}

El primer trabajo del que me ocuparé es Toponimia sevillana. Ribera, Sierra y Aljarafe de María Dolores Gordón Peral (1995). Una investigación realizada desde la tradición de la filología hispánica que se enfoca en los nombres de lugar de, aproximadamente, "la mitad septentrional de la Provincia de Sevilla” (Gordón 1995: 37), un área que se extiende desde la llanura ribereña del Guadalquivir hasta el Aljarafe sevillano. Aunque la autora no se preocupa por explicar cuál es su objeto de estudio, sí realiza una descripción de la materia con que trabaja, que se conforma por todos los nombres de lugar circunscritos al área designada. Asimismo, da a conocer que, debido a la magnitud del corpus, lleva a cabo la siguiente selección:

[...] hemos decidido restringir el campo de acción a los casos de más interés para la Lexicología, la Dialectología, la Historia de la Lengua, la Onomástica, la Historia o la Arqueología, es decir, atendiendo de preferencia a un criterio lingüístico, pero sin olvidar aspectos no lingüísticos en modo alguno exentos de importancia en un área tan peculiar como es la toponimia. (Gordón 1995: 55)

La observación de estos elementos a partir de la diacronía está evidentemente condicionada por el interés de Gordón por el pasado de estos nombres y, con esto, el pasado de la lengua y de 
sus hablantes. Tal y como lo señala cuando describe algunas de las características de su materia de estudio.

Como es sabido, los nombres de lugar se caracterizan por la función casi exclusivamente referencial (la de señalar un lugar) que ejercen. Tal función referencial (en la que consiste el principal rasgo diferenciador de los nombres frente a los apelativos, en los que suelen basarse originariamente) lleva consigo una acusada tendencia a la pérdida parcial o completa de conciencia de un contenido semántico para los hablantes. (Gordón 1995: 30)

La cita anterior señala la historicidad como factor inherente para el análisis de cualquier nombre de lugar. Es decir, aunque estas voces tienen cierta tendencia hacia la pérdida del contenido semántico inicial por parte de sus hablantes, se entiende que dentro de su estructura más arcaica se encuentran las pistas del que fuese su significado original. De esta manera, la autora procede a señalar el objetivo principal de su estudio: "está claro que es menester pensar en un método que nos encamine a la consecución del objetivo: lograr la interpretación más certera de los nombres - interpretación etimológica, semántica” (Gordón 1995: 31) [El énfasis es mío]. El objeto de estudio que le interesa, entonces, tendría que ser el significado original de cada una de las voces con que trabaja. Aquí, la interpretación puede entenderse como el fin de un recorrido a través de distintas capas históricas hasta llegar al significado más antiguo que sea posible plantear para cada nombre.

En este caso, la metodología propuesta requiere, antes que nada, la recopilación de una gran cantidad de información lingüística y extralingüística que permita este viaje en el tiempo. El conjunto de la información, tal y como explica Gordón (1995), debe seguir las siguientes directrices: 1) facilitar la recuperación de las formas históricas y actuales con que se registra el topónimo en análisis; 2) facilitar la descripción del contexto extralingüístico (sea este de tipo arqueológico, histórico o geográfico) al que el nombre puede hacer referencia; y 3) facilitar el contraste entre las formas apelativas y onomásticas (tanto actuales como históricas) dentro de la 
zona investigada y otras áreas hispánicas. Una vez reunida esta información, el siguiente paso debe ser el planteamiento de una base etimológica para cada nombre, que se ajuste a la representación fonética de una lengua específica (sea o no el español). Este ejercicio colaborará con la tarea de restituir el valor semántico y referencial inicial de la palabra. Nótese, entonces, que la recuperación del étimo formal (o la forma fónica) es solo un paso previo y necesario para aproximarse a un objeto más amplio, lo que indica que el interés de Gordón no es, precisamente, la reconstrucción de lema (como estructura o signo lingüístico), sino únicamente de su significado léxico. Ahora, es interesante mencionar que la propia autora señala los límites del método propuesto.

[...] con demasiada frecuencia, tales elementos no permiten una interpretación completamente satisfactoria ni siquiera para los más destacados especialistas, por no existir una base documental realmente sólida, de modo que hemos de contentarnos en más de una ocasión con una hipótesis. (Gordón 1995: 31)

En otras palabras, en aquellas oportunidades donde la información al alcance de la mano no sea la suficiente para plantear un étimo coherente, bastará con describir aquellas pistas que el investigador se permita conceder a partir de su propia subjetividad sobre los conceptos y normas sociales que involucran a cada caso específico y que, precisamente, desconoce. Solo en estos ejemplos la autora califica la interpretación de hipotética, aunque esto no necesariamente guarde afinidad con las propiedades semánticas del objeto de estudio que dice interesarle. La interpretación del significado de una palabra, más allá del momento histórico de interés, supone adentrarse en un saber social, que se construye mediante el acto epistémico de la intuición, es decir, un saber que solo puede tener validez a partir de la intersubjetividad. La aproximación subjetiva, en palabras de Itkonen (2008), no podría ser válida o no válida, ya que solo es subjetiva y las estructuras de conocimiento lingüístico se hacen reales en la intersubjetividad, en la concretización de una norma. Por tanto, siempre debe ser visto como un planteamiento hipotético. 
Las etimologías formuladas, más allá de la evidencia textual que las ampare, se darán necesariamente en calidad de hipótesis, y aquellos planteamientos que no tengan el respaldo documental suficiente deben ser asumidos como conjeturas guiadas por la introspección y, en menor medida, por la intuición.

Gordón (1995) presenta, indistintamente, ambos tipos de resultados (voces con una solución etimológica y sin ella) junto con una elaborada descripción de los elementos utilizados para el análisis de cada nombre, entre ellos, por ejemplo, su locación geográfica, otros apelativos similares en la zona, la pronunciación y el significado léxico que los actuales hablantes utilizan para cada topónimo, y la referencia de todos los textos utilizados para el estudio de estas voces. Así, debido a la organización de los datos y las características primordialmente históricas de la documentación consultada por la autora, pareciera que se está trabajando con un objeto distinto. Puede que, más bien, la principal particularidad del objeto sea de índole historiográfica. Así, el objetivo principal del texto sería de tipo documentalista, y el objeto, la historiografía de cada uno de los topónimos seleccionados. ${ }^{3}$

Por ejemplo, nótese cómo Gordón (1995) inicia la descripción del topónimo Alanís, en la sección destinada al estudio de nombres de municipios:

Tratando de la historia de la localidad de Alanís, manifiestan los autores del Catálogo Arqueológico:

«El primer documento que conocemos donde aparece nombrado Alanís es un libramiento de 1392 al Consejo de Sevilla [...] siendo ya frecuentes las citas a partir de la fecha mencionada»

Aquí adelantamos la fecha de la primera documentación del topónimo mayor, pues lo hemos encontrado ya hacia 1344, en el Libro

\footnotetext{
3 Aunque Gordón (1995) también presenta algunos apartados donde da a conocer ciertas estructuras morfológicas recurrentes en la toponimia observada (1995: 491500), la mayoría de las veces son vistas solo como apéndices con información que puede ser de utilidad a quien se interese en temas referidos al sistema morfosintáctico de los nombres de lugar.
} 
de la Monteria de Alfonso XI, formando parte de un antropónimo, Pasqual Gil de Alanjs, y, más tarde, en 1384 y 1386, en otro antropónimo -Alonso Gil de Alanis, probablemente hijo o pariente, al menos, del personaje citado por Alfonso XI-, y ya propiamente como nombre de población. De otro lado, el aparato documental ratifica el hecho de que con posterioridad al siglo XIV se cita el lugar con mucha mayor frecuencia e inevitablemente con forma Alanís, hecha la salvedad del texto fechado en 1563, donde la grafía al anís tal vez haya sido motivada por etimología popular [...]. (Gordón 1995: 59)

No es poco importante - la autora se encarga de recordarlo- la documentación histórica que existe del nombre en análisis. Parece quedar claro que no se puede dejar de lado cómo el tipo de acceso que se tiene sobre el dato influye también en la manera de plantear los objetivos y metodología de la investigación. Cuando la autora describe la etimología de la palabra, una vez asumida la base árabe, señala brevemente la posible estructura interna de la palabra, apoyándose en documentos lexicográficos como el diccionario de Corominas, y en las particularidades geográficas e históricas de la zona.

[...] y esto, dada la característica configuración del nombre a base del artículo ár. al. que acompaña de modo inherente a los apelativos arábigos según el tratamiento del romance popular (11). Por lo que se refiere al segundo componente, anis, puede interpretarse como el nombre árabe de la planta que en castellano antiguo se denomina matalabuva (12), o bien como el adjetivo ár. ani sun 'dulce, sociable, afable', de la misma raíz e incluso cuasi homónimo del primero, el sustantivo ani sun 'anís, planta' (13). Con todo, quizá haya que inclinarse por un original fitónimo, dada la marcada representatividad de este tipo de nombres en la zona serrana (14). (Gordón 1995: 60-61)

Aquí, la explicación sobre la estructura del nombre no necesita de un análisis comparado entre textos que ratifiquen cuál es su composición interna o el sentido con que la voz ha sido utilizada. El hecho de poseer una amplia documentación lexicográfica y, en general, textual, parece hacer que el foco de la explicación se traslade de su 
estructura morfosintáctica hacia un eje documental, más relacionado con la lexicografía histórica. Por ejemplo, al trabajar el topónimo Alcalá del Río, en la misma sección, Gordón (1995) asume directamente la base árabe al-qalat 'castillo' sin una demostración sobre el porqué de esta. Luego, para explicar su referencia histórica, resume las conclusiones de distintos estudiosos de la toponimia arábiga y determina que debió ser una "denominación administrativomilitar y al mismo tiempo topónimo genérico, [que] a un núcleo de importancia considerable y con unas defensas que responde al tipo mayor de construcción militar” (Gordón 1995: 63). En cuanto al segundo componente, del Rio, a través del examen documental, señala que "aparece ya formando parte de los topónimos en los primeros documentos castellanos, en los que se describe, seguramente, para mayor especificación, para mejor distinguirla de la otra Alcalá [...]"(Gordón 1995: 63). Para finalizar, como también lo hace en el primer topónimo mencionado, da a conocer una lista extensa de la documentación total que se tiene hasta la fecha de dicho lema:

Documentación. 1253: Alcalá del Río; “A Pero, VI yugadas, en Alcalá del Río" (Repartimiento II, pp. 16, 19, 54, 56, 57 [...]). 1253: "Estos heredan la heredat del pan en Alcalá de Guadalquiuir"; "en Guillena, aldea de Alcalá de Guadalquinir" [...] 1280: "et poso sobre Alcalá la que dicen del Río, que es y cerca de Senilla", "et de commo enbio çercar Alcalá del Río et le tomo por fuerça" [...] 1506: "consejo de Alcalá del Río" (Donadíos, p. 61, nos 294, 295) [...]. (Gordón 1995: 64)

De otro lado, la propuesta metodológica de Gordón (1995), que plantea un objeto de estudio etimológico - semántico, organiza la presentación del corpus según el mismo criterio.

[...] puesto que el método onomástico que seguimos se encamina principalmente a la restitución de la forma y del contenido (semántico o histórico) iniciales de los nombres de lugar-entendiendo por 'iniciales' los que tenían en el momento mismo de la imposición, y no los del étimo al que como apelativos pudieran remitir-, clasificamos estos según la adecuación de su contenido a uno u otro campo [semántico]. (55-56) 
La autora organiza todas las voces según la motivación semántica de su étimo. Así, por ejemplo, se proponen categorías como 'Orónimos y denominaciones del relieve del terreno' o 'Hidrónimos'. Este tipo de clasificación, según Gordón, será útil al momento de ahondar en una estructura cognitiva generalizada que responda al acto de nombramiento de objetos geográficos por parte de cualquier comunidad (Gordón 1995: 30). Tanto es así que se anima a proponer dos procesos sistematizados que se dan durante la denominación: a) aquellos que tienen como principal motivación la referencia a un objeto propio de la realidad física, bien sea por criterios topográficos o actividades productivas llevadas a cabo en el lugar; y b) aquellos que poseen como mayor motivación semántica la historia circunstancial correspondiente a los hablantes. El mencionado criterio de organización, sin embargo, parece ratificar todavía más un objeto de características historiográficas. Puesto que se estaría presentando, más bien, un mapa de las características referenciales del paisaje para un área específica en el pasado, antes que una propuesta sistemática sobre el modo de concebir el espacio por parte de los antiguos hablantes en la zona.

2.2. Morfemas arcaicos en la toponimia centro-andina: los sufijos $-y,-n$ y $-r a$

El siguiente texto por comentar proviene también de una tradición filológica, aunque dedicada al análisis de lenguas andinas, en este caso del aimara. La obra Voces del Ande de Cerrón-Palomino (2008a) reúne un conjunto de ensayos sobre onomástica andina, cuyo enfoque radica, principalmente, en lenguas como el quechua o el aimara, aunque sin dejar de lado otras también presentes en la región como el puquina, el uro-chipaya y el mochica. De todos estos, se ha seleccionado el texto que tiene como título "Morfemas arcaicos en la toponimia centro-andina: los sufijos -y, -n y -ra” porque en este, a diferencia del estudio de Gordón (1995), no se persigue la interpretación de los nombres de lugar, sino de las estructuras que se encuentran en su interior. 
En este caso, Cerrón-Palomino (2008c) no circunscribe los datos seleccionados a un área específica del territorio andino, por el contrario, parece indicar que, del total de la materia de estudio que le compete (sea toda la toponimia asociada a los Andes sudamericanos), tomará en cuenta los datos que le sean más pertinentes. Es decir, todos aquellos que, a su propio juicio, contengan las estructuras morfológicas que le interesan estudiar.

En la presente nota nos ocuparemos del estudio de tres sufijos recurrentes en la toponimia de los Andes centro-sureños. Tales desinencias, cuya etimología $<<$ descubriremos $>>$ a partir de la información toponímica, no han sido estudiadas aún, ni siquiera por los aficionados de ayer y hoy (que abundan), puesto que, entre otras razones, su identificación a partir de la información lingüística colonial y moderna resulta poco menos que imposible cuando no se las aborda desde una dimensión diacrónica que tome en cuenta, además, el contacto idiomático, milenario y profundo, entre las dos «lenguas mayores» del antiguo Perú: el quechua y el aimara. (Cerrón-Palomino 2008c: 193) [El énfasis es mío]

Si, como se dijo en el apartado anterior, el objetivo de Gordón (1995) apuntaba a un objeto historiográfico —un proyecto de lexicografía en el que la historia de la lengua y la de sus hablantes toman igual importancia para documentar el recorrido semántico y textual de la toponimia de toda una región-, Cerrón-Palomino (2008c: 191-208) tiene como principal finalidad explicar un grupo de estructuras morfológicas correspondientes a la lengua aimara, tal y como se observa en la cita anterior. En este sentido, quizás por la relativa escasez de fuentes documentales, la selección del dato tiene como principal motivación la estructura y motivación semántica antes que el espacio geográfico.

(a) Configuraciones naturales:
Anta-ra-y <anta 'cobre'
(Q/A), (Paucartambo)
Anda-ra-y <anta'cobre'
(Q/A), (Condesuyos) [...]
Huanca-ra-y <wanka'peñon'
(Q/A), (Angares,
Andahuailas, Cotabambas) 
(b) Edificaciones:
Cancha-ra-y <kanĉa 'cerco'
(Q/A), (La Unión)
Chaca-ra-y <čaka 'puente'
(Q/A),(Parinacochas, Chum- bivilcas) [...] (Cerrón-Palomino 2008c: 203)

Si bien cada uno de estos nombres procede de distintas regiones de los Andes peruanos, el foco de atención es otro. Los objetos de estudio con que trabaja son estructuras morfológicas que forman parte de la historia de esa lengua y las hipótesis que el autor plantea buscan la reconstrucción de su forma etimológica. Asimismo, como Gordón (1995), se detiene en la necesidad de mantener una mirada histórica frente al corpus y, por tanto, sugiere un método de análisis que debe tomar en cuenta toda la información pertinente (sea esta del tipo sincrónico o diacrónico).

En este mismo sentido, Cerrón-Palomino plantea un esquema de trabajo en el que deja entrever a qué se refiere cuando se habla de descubrir la etimología de las estructuras lingüísticas mencionadas.

En lo que sigue, procederemos con el estudio de las desinencias exclusivamente toponímicas $-y,-n$ y $-r a$, de acuerdo con el siguiente esquema: (a) ilustración del sufijo; (b) su caracterización morfológica; (c) su etimología formal y semántica... (Cerrón-Palomino 2008c: 194) [El énfasis es mío]

La propuesta señala los pasos que el autor cree necesarios para poder aproximarse a la realidad objetiva de estos sufijos. Primero, (a) se debe comprender que son estructuras que aparecen en la toponimia andina y que, por lo general, están representadas por las grafías $\langle-\mathrm{y}\rangle,<-\mathrm{n}\rangle,<-$ ra $>$; en segundo lugar, (b) se debe entender el comportamiento tipológico del morfema, en este caso, por ejemplo, que <-y> se añade siempre a bases nominales; $y$, en tercer lugar, (c) se debe asumir que la reconstrucción etimológica de la estructura comprende tanto una aproximación a la representación fonética, como a su significado original. Entonces, para alcanzar las estructuras de naturaleza social que el autor plantea como objetos, se requiere de la reconstrucción de una realidad fónica y semántica. 
De otro lado, la metodología que utiliza el autor para cumplir con este propósito no resulta del todo diferente a la sugerida por Gordón (1995), ya que ambos conciben un objeto de características históricas. Sin embargo, más allá del uso de fuentes sincrónicas y diacrónicas, Cerrón-Palomino (2008c) también recurre a saberes científico-teóricos generados dentro del mundo 3 del modelo popperiano. ${ }^{4}$ Así, por ejemplo, cuando propone la representación de lo que él llama etimología formal, utiliza reconstrucciones morfológicas hipotéticas pertenecientes a las protolenguas del quechua y el aimara. ${ }^{5}$ Como él mismo indica, es un tipo de práctica necesaria en un contexto donde la documentación escrita, a diferencia del ámbito estrictamente hispánico, es escasa.

El conocimiento de los cambios operados en las proto-lenguas respectivas [en este caso tanto el quechua como el aimara], y postulados por el lingüista en calidad de reglas sistemáticas, no solo ayuda a resolver en forma ordenada los problemas de interpretación toponímica sino también, además de confirmar hipótesis previas, posibilita detectar de manera efectiva relaciones y asociaciones entre entidades que, de otro modo, burlarían inclusive la atención del propio especialista. (Cerrón-Palomino 2008b: 163)

\footnotetext{
4 Como explica Henríquez Garrido (2004), aquí se encuentra una de las utilidades de esta realidad ontológica, pues "la importancia que tiene la autonomía del mundo 3 radica en la posibilidad de hacerse descubrimientos en él, del mismo modo en que se llevan a cabo en el mundo físico. Es decir, que si bien el mundo de las ideas y del conocimiento objetivo es producto de la mente humana, este orden de los inteligibles y de lo simbólico genera otros problemas y pensamientos sin que ningún otro sujeto intervenga en su producción. En otros términos, se puede decir que el mundo 3 trasciende a las mentes que lo crearon, desbordando las aportaciones iniciales de las que partieron, con un número indefinido de nuevos problemas y de nuevas soluciones" (2004: 120).

${ }^{5}$ Un ejemplo se observa al plantear la etimología del sufijo <-y $>$, pues se presume que provendría de la forma protoaimara /*-wi/ y cambiaría a la forma /-y/ dentro de la toponimia quechua (Cerrón-Palomino 2008c). Se trata de un sufijo que se hace productivo frente a verbos y raíces ambivalentes, como indica Cerrón-Palomino (2008c), aunque la toponimia andina también refleja distintos ejemplos en los que se añade a una base nominal y todavía mantiene su funcionalidad, de manera que denota contenidos como 'lugar donde suele realizarse $\mathrm{X}$ '.
} 
El conocimiento sobre estas protoformas, que deben tomarse siempre en calidad de hipótesis, supone un primer paso necesario antes de establecer cualquier relación acerca de un significado. Así, en el caso del texto referido, una vez formulada la hipótesis con respecto a la forma etimológica de cada sufijo, la propuesta de la etimología semántica para cada uno de estos elementos se realiza a partir del examen minucioso de diferentes documentos coloniales y republicanos, así como de estudios léxicos o gramaticales referidos a las lenguas andinas. Por ejemplo, cuando se explica el caso de cotaray $\sim$ quta-ra-y $\sim$ quta-ra-wi*:

[...] creemos que el sufijo -ra de los topónimos listados al principio tiene una evidente relación, en forma y significado, con la porción -ra de la desinencia registrada por Bertonio [cuya descripción lexicográfica y gramatical del aimara data de 1603]. El hecho de que esta aparezca soldada a $<$ cta $>$, sufijo que tenía existencia propia, y que también, gracias a él, $<<$ los nombres o adjetivos [...]significan multitud de cosas o personas, que son lo que el nombre significa $>>$ (cf. op. cit. 262), nos induce a la sospecha de que, por la época en que el jesuita introduce el complejo desinencial <-cta-ra>, la segunda porción de este ya se iba tornando obsoleta, uno de cuyos efectos habría sido la pérdida de su capacidad combinatoria con un radical nominal $[\ldots]$

De aceptare la hipótesis adelantada, y creemos que hay buenas razones para ello, ahora estaríamos en condiciones de sostener que -ra, en los topónimos listados al comienzo, significaría 'abundancia o multitud de $\mathrm{X}$ ', donde $\mathrm{X}$, como sabemos, vale para el referente expresado en el radical. D modo que [...] la diferencia entre Cota-y y Cota-ra-y, radicaría en que el primero equivale solamente a 'lugar donde existe laguna', mientras que el segundo estaría significando 'lugar donde existen muchas lagunas'. (Cerrón-Palomino 2008c: 206)

El autor busca, entonces, adentrarse en un tipo de conocimiento social, la estructura de una norma determinada, y para hacerlo se apoya no solo del conocimiento de la lengua, sino también en un tipo de saber científico (que es también social) y un manejo eficiente de los datos que dispone. De esta manera, la descripción del 
objeto de estudio y los objetivos planteados por el autor guardan coherencia con el tipo de metodología, tanto en la propuesta de una representación fónica como en la interpretación de un significado etimológico.

2.3. Columbia Plateau Indian Place Names: What can they teach us?

El último de los casos que se ha tomado en cuenta es el artículo de Eugene Hunn (1996), "Columbia Plateau Indian Place Names: What can they teach us?”, en el que se estudian más de 1000 topónimos de la lengua Sahaptin. La materia de estudio que este autor toma en consideración comprende toda la toponimia ubicada en la meseta de Columbia (Estados Unidos) que pueda ser asociada con el Sahaptin. ${ }^{6}$ La selección de los datos por analizar se puede apreciar en la siguiente cita del autor:

The present report is an analysis of the extant corpus of Sahaptin place-names, highlighting features of the indigenous geographic naming system both peculiar to this language and culture and shared with other systems with which the Sahaptin data may be compared. (Hunn 1996: 4) [El énfasis es mío]

El corpus recogido por Hunn ${ }^{7}$ está compuesto, entonces, por aquellos nombres representativos del sistema referencial geográfico del Sahaptin que, al mismo tiempo, comparten ciertos elementos con otros sistemas lingüísticos. El principal motivo de esta selección se debe a que el autor está interesado en un objeto de características etnográficas, pues espera aproximarse al modo en que una comu-

6 El Sahaptin es, en realidad, una etiqueta lingüística que agrupa un conjunto de dialectos mutuamente inteligibles que se hablan en los actuales territorios de Washington y Oregon por poblaciones indígenas que residen en las riveras sureñas del río Columbia desde los primeros contactos con colonos europeos (Hunn 1990).

7 A diferencia del corpus que recogen Cerrón-Palomino (2008c) y Gordón (1995), intenta representar un contraste con otros sistemas lingüísticos con que el Sahaptin haya podido tener algún tipo de contacto o convivencia. En otras palabras, mientras que a Cerrón-Palomino le interesan estructuras del sistema del aimara y a Gordón, el pasado de la toponimia hispánica, a Hunn (1996) le parece interesar el contraste entre los sistemas de nombramiento de lugares que han convivido en la meseta de Columbia. 
nidad entiende y organiza su territorio al comparar la forma en que distintos sistemas lingüísticos dan referencia del mismo espacio geográfico.

I would like to explore here the potential for a comparative ethnosemantic analysis of place-name systems. Cognitive anthropologists have pursued systematic cross-language comparisons in such semantic domains as kinship relations, color categories, and folk biological taxonomies. These studies have demonstrated the existence of widespread, if not universal, patterns that structure human undertanding within these domains (e.g., Atkins 1974; Berlin 1992; Kay, Berlin, and Merrifield 1991) Such studies have provided significant support for modular theories of cognitive processing (Hischefeld and Gelman 1994) and help define the 'natural' cognitive foundation with respect to which cultural diversity and individual creativity may be more fully appreciated. (Hunn 1996: 4) [El énfasis es mío]

Como es posible apreciar en la cita anterior, el autor explica que, de la misma manera en que es posible hablar de categorías semánticas relacionadas con el color o las relaciones de parentesco, también debería poderse sostener la existencia de una categoría relacionada con la organización espacial de la geografía. Levinson y Burenhult (2008) explican que este tipo de perspectiva intenta responder a las siguientes preguntas: (1) ¿cómo se divide el paisaje en distintas categorías mentales y cómo son nombradas?, (2) ¿existen diferencias en la manera en que se organizan estas categorías cuando se comparan sistemas lingüísticos que han convivido en una misma región y que no han estado necesariamente en una situación de contacto? y (3) ¿Puede esto ayudar a entender el modo en que funciona la categorización mental del paisaje?. ${ }^{8}$ El objeto de estudio de Hunn (1996) es, por lo tanto, la cognición del espacio geográfico de una

8 Traducido del inglés desde Levinson y Burenhult: "Initially targeting the formal and functional properties of place names, the research agenda was widened to also incorporate the linguistic categorisation of the physical environment, as reflected in generic landscape terms. The general questions serving as guidelines for the research can be summarised as follows: 
comunidad, es decir, las estructuras sistemáticas que la gobiernan. Así, aunque puede decirse que los procesos cognitivos se realizan de manera individual y subjetiva, la meta que se intenta alcanzar es el acuerdo tácito que una comunidad determinada elabora a partir de la cognición. En este sentido, Hunn propone un objeto cuya naturaleza corresponde al mundo 3 popperiano, pues es el resultado de una realidad conceptual, en la que se ubican las normas sociales (Itkonen 2008). De modo que el planteamiento de un objeto de estas características lo motiva a proponer los siguientes objetivos:

A primary goal of ethnoscience is to abstract general principles of classification and nomenclature from comparative studies of whole semantic domains in a variety of languages and cultures and then to understand cultural diversity against that universalist background [por antecedente universalista se refiere a las categorías semánticas supuestamente universales del pensamiento, como las relaciones de parentesco]. (Hunn 1996: 6)

El mismo Hunn (1996) indica que la suya supone un tipo de investigación que se puede situar dentro la etnografía, disciplina cuyo principal objetivo es el análisis de las semejanzas y diferencias entre el pensar y el actuar de una comunidad, operaciones en las que el lenguaje cumple un rol indispensable. Así, sus principales objetivos son la abstracción de los patrones sistemáticos con que distintas comunidades organizan el mismo paisaje geográfico a través de su lengua, para encontrar semejanzas y diferencias que le permitan plantear o negar la existencia de estructuras universales que gobiernan el pensamiento.

El método que Hunn encuentra más apropiado para las características de su objeto utiliza la elicitación de nombres de lugar

1. How is landscape divided into categories, and how are these categories named? Are there cross-linguistic differences in how landscape is divided into categories? Which are the main determinants of landscape categorisation?

2. How do we formally identify place names? What places are place names employed to refer to? How are places semantically construed for this purpose?

3. What is the denotational relation between landscape terms and place names?" (2008: 140). 
como su principal herramienta, además del registro escrito pertinente (sincrónico o diacrónico). Es decir, en su caso, no se puede prescindir del trabajo de campo por parte del investigador, ya que, conjuntamente con la recopilación de datos lingüísticos, necesita de la descripción de distintos elementos extralingüísticos, como los referentes geográficos y biológicos de la región, las prácticas productivas de la comunidad, o la descripción topográfica del espacio referido (Hunn 1996: 6). Este tipo de datos le permiten al autor observar la relación entre las estructuras lingüísticas de una lengua y las categorías semánticas asociadas con el espacio. Por este motivo, en el caso de la lengua Sahaptin, organiza la presentación de sus resultados a partir de la relación entre motivaciones semánticas (como referentes topográficos, biológicos o metafóricos) y estructuras sintácticas o morfológicas del Sahaptin (Hunn 1996: 15-17). Por ejemplo, menciona que una de estas categorías semánticas corresponde a los fenómenos topográficos, que pueden ser divididos entre terrestres o hidrográficos, como, por ejemplos, litorales o elementos acuáticos (Hunn 1996: 16). El autor, además, relaciona estas clases referenciales con las categorías gramaticales correspondientes a las raíces de diferentes formaciones lingüísticas que se utilizan como nombres de lugar. Tal y como lo podemos observar en la tabla 1.

El método utilizado para el análisis y organización de los datos, en efecto, se adecua con el objeto de estudio que propone nuestro autor. Debido a que sus resultados intentan presentarse como la sistematización hipotética de estructuras sintácticas y morfológicas en contraste con otras del tipo semántico. La toponimia es vista aquí, entonces, como un todo, como la representación de un sistema cognitivo con el que una comunidad específica organiza espacialmente el espacio en el que habita. 
Tabla 1. (Hunn 1996: 17)

Sahaptin topographical place names

\begin{tabular}{|c|c|c|c|}
\hline & \multirow[b]{2}{*}{$\begin{array}{l}\text { Terrestrial } \\
\text { (65 cases) }\end{array}$} & \multicolumn{2}{|c|}{ Hydrographic } \\
\hline & & $\begin{array}{l}\text { Shoreline } \\
\text { (61 cases) }\end{array}$ & $\begin{array}{l}\text { Aquatic } \\
\text { (58 cases) }\end{array}$ \\
\hline $\begin{array}{l}\text { Adjectival } \\
\text { (45 cases) }\end{array}$ & $\begin{array}{l}\text { q'tit-aas } \\
\text { 'hard place' } \\
\text { maqát } \\
\text { 'yellowish' }\end{array}$ & $\begin{array}{l}\text { kis } \\
\text { 'stinking' } \\
\text { lik'á } \\
\text { 'bent' }\end{array}$ & $\begin{array}{l}\text { k'ttu-lí } \\
\text { 'swift one' } \\
\text { muk-múk } \\
\text { 'muddy' }\end{array}$ \\
\hline $\begin{array}{l}\text { Nominal } \\
\text { (70 cases) }\end{array}$ & $\begin{array}{l}\text { túli } \\
\text { 'cave' } \\
\text { q'mit } \\
\text { 'rocky defile' }\end{array}$ & $\begin{array}{l}\text { p'na 'rock bowl } \\
\text { carved by } \\
\text { current' } \\
\text { amá 'island' }\end{array}$ & $\begin{array}{l}\text { q'up } \\
\text { 'waterfall' } \\
\text { watám-pa } \\
\text { 'at lake' }\end{array}$ \\
\hline $\begin{array}{l}\text { Verbal } \\
\text { (69 cases) }\end{array}$ & $\begin{array}{l}\text { wa-lúuk } \\
\text { '[land] soars up } \\
\text { like bird' } \\
\text { tawayawish-nmi } \\
\text { 'of landslide' }\end{array}$ & $\begin{array}{l}\text { pá-wan-kyuut } \\
\text { 'rivers join' } \\
\text { tama-wáyk-ash } \\
\text { '[rocks] laid across } \\
\text { place' }\end{array}$ & $\begin{array}{l}i \text {-múti'k-sha } \\
\text { 'it bubbles' } \\
\text { wápay- } k-t \\
\text { 'water accelerates } \\
\text { downward' }\end{array}$ \\
\hline
\end{tabular}

\section{A manera de conclusión}

A lo largo de estas páginas se ha intentado establecer la diferencia entre la materia y el objeto de estudio de tres investigaciones concernientes al análisis de la toponimia. Cada una de estas está condicionada según las perspectivas de la rama de la lingüística a la que pertenecen. Así, primero, se tomó en cuenta dos perspectivas filológicas, una interesada en el ámbito hispánico de Andalucía y la otra, en las lenguas que se encuentran en el territorio de los Andes americanos. La tercera, de otro lado, corresponde a la perspectiva de la lingüística cognitiva, y se interesa en las tipologías del pensamiento.

En el caso de Gordón (1995), el principal interés se dirige al estudio historiográfico de los nombres de lugar $\mathrm{y}$, en aquellos casos en que la documentación se lo permite, propone una hipótesis acerca de su significado léxico primigenio. Cerrón-Palomino (2008c), por otro lado, se aproxima a un objeto de características 
sistemáticas, pues le importan algunas estructuras, propias de un saber social, que pertenecen a la historia de la lengua aimara. Por último, Hunn (1996), cuyo interés es de tipo etnográfico, construye como objeto de estudio la cognición sistematizada que una comunidad posee acerca de su espacio geográfico.

A pesar de que los tres textos que han sido analizados se enfocan en materias de estudio similares, existe un proceso de reconocimiento y construcción teórica que plantea objetivos y objetos de estudio diferentes, más allá de que los autores sean o no conscientes de esto. No basta con señalar que la materia de estudio es la toponimia de determinada zona o de determinada lengua, existe también un proceso en el que el investigador interactúa con ella, la analiza y conceptualiza para definir con claridad qué es lo que quiere de ella. Los objetivos e intereses con respecto a determinada materia de estudio frecuentemente son guiados por la disciplina teórica en la que descansa el ejercicio y es a partir de la misma que se inicia la construcción del objeto. Dicho de otro modo, la materia de estudios, o lo que concebimos por esta, puede tener dentro de sí diferentes objetos de análisis según la elaboración teórica con que se la procese. La demostración desde la toponimia debería ser también plausible para otras ramas de la lingüística y, así, intentar entender de manera más adecuada cuál es el rumbo que ha tomado la disciplina. No solo eso, al ser conscientes de la naturaleza de nuestro objeto de estudio será más probable que las metodologías que seleccionemos para su análisis sean las más adecuadas y acordes a nuestros objetivos. De una u otra manera, debería ser inevitable caer en esta clase de razonamiento, en especial cuando se trata de una ciencia que busca concebir la realidad de la lengua, construcción que, a fin de cuentas, existe como una hipótesis. 


\section{Referencias bibliográficas}

Cerrón-Palomino, Rodolfo

2008a Voces del Ande. Lima: Fondo Editorial de la PUCP.

2008b "La naturaleza probatoria del cambio lingüístico: a propósito de la interpretación de la toponímica andina”. En CerrónPalomino 2008a: 163-180.

2008c "Morfemas arcaicos en la toponimia centro-andina: los sufijos $-y,-n$, y -ra”. En Cerrón-Palomino 2008a: 193-208.

Cerrón-Palomino, Rodolfo y otros

1983 Guia para estudios de toponimia. Lima: Universidad Nacional Mayor de San Marcos, Centro de Investigación de lingüística aplicada.

Coseriu, Eugenio

1973 Sincronía, diacronía e historia. Madrid: Editorial Gredos.

FERnÁndez Pérez, Milagros

1986 La investigación lingüística desde la filosofía de la ciencia ( $A$ propósito de la lingüística chomskiana). Santiago de Compostela: Universidad de Santiago de Compostela, Servicio de Publicaciones.

Frago, Juan

1991 "Problemas, Métodos y enseñanzas de la toponimia". Actas de las I Jornadas de Onomástica, Toponimia. Vitoria-Gasteiz: Euskaltzaindia.

Gordón, María Dolores

1995 Toponimia Sevillana. Ribera, Sierra y Aljarafe. Sevilla: Diputación Provincial de Sevilla, Fundación Luis Cernuda.

2011 "La memoria de los lugares: la toponimia". Revista ph. Instituto Andaluz del Patrimonio Histórico. 77, 90-91.

Henríquez Garrido, Ruy

2004 "La teoría de la mente objetiva de Popper". En Hipótesis y verdad en ciencia: ensayos sobre la filosofía de Karl R. Popper. Ed., A. Rivadulla . Madrid: Editorial Complutense, 115-124.

HunN, Eugene

1990 "Ncb' $i-W d n a,<<$ The Big River $>>$ : Mid-Columbia Indians and Their Land”. Seattle: University of Washington Press. 
1996 "Columbia Plateau Indian Place Names: What can they teach us?”. Journal of Linguistic Anthropology. 6, 1, 3-26.

ITKONEN, Esa

2008 ¿Qué es el lenguaje?: introducción a la filosofía de la lingüistica. Traducción y edición española a cargo de Araceli López Serena. Madrid: Biblioteca Nueva.

KARI, James

1989 "Some principles of Alaskan Athabaskan toponymic knowledge”. En General and Amerindian ethnolinguistics. Berlín: Mouton, 129-150.

LEVINSON, Stephen

2003 Space in language and cognition. Explorations in cognitive diversity. Londres: Cambridge Univesity Press. https://doi. org/10.1017/cbo9780511613609

LeVinson, Stephen y Niclas Burenhult

2008 "Language and landscape: A cross-linguistic perspectiva”. En Language Sciences. Nijmegen: Language and Cognition, Max Planck Institute for Psycholinguistics, 136-150. https://doi. org/10.1016/j.langsci.2006.12.028

López Serena, Araceli

2013 "Variación y variedades lingüísticas. Un modelo teórico dinámico para abordar el estatus de los fenómenos de variación del español hablado en Andalucía". En Conciencia y Valoración del habla andaluza. Ed., Antonio Narbona. Sevilla: Universidad Internacional de Andalucía, 73- 127.

Rodríguez Adrados, Francisco

2011 "Hacia una teoría de la ciencia toponímica”. Revista Española de Lingüistica. 32, 1, 33-51.

SENFT, Gunter

2008 "Landscape terms and place names in the Trobriand Islands - the Kaile'una subset”. En Language Sciences. Nijmegen: Language and Cognition, Max Planck Institute for Psycholinguistics, 340-361.https://doi.org/10.1016/j.langsci.2006.12.001

Trapero, Maximiano

1995 Para una teoría lingüistica de la toponimia: estudios de toponimia canaria. Las Palmas de Gran Canaria: Universidad de Las Palmas. 
1996 “Sobre la capacidad semántica del nombre propio". El Museo Canario (Las Palmas de Gran Canaria). LI, 1996, 337-353.

VAN LANGENDONCK, Willy

2008 Theory and tipology of Proper Names. Berlín: Walter de Gruyter $\mathrm{GmbH} \&$ Co.

ZAMBOnI, Alberto

1988 La Etimología. Madrid: Editorial Gredos.

Recibido: $10 / 03 / 2016$

Aceptado: 18/08/2017 